\title{
PDI/PDLI inhibitors for the treatment of advanced urothelial bladder cancer
}

This article was published in the following Dove Press journal:

OncoTargets and Therapy

\author{
David D Stenehjem ${ }^{1,2}$ \\ Dao Tran' \\ Michael A Nkrumah' \\ Shilpa Gupta ${ }^{2,3}$ \\ 'Department of Pharmacy Practice \\ and Pharmaceutical Sciences, College \\ of Pharmacy, University of Minnesota, \\ 2Masonic Cancer Center, University of \\ Minnesota, ${ }^{3}$ Division of Hematology, \\ Oncology and Transplantation, \\ Masonic Cancer Center, University of \\ Minnesota, Minneapolis, MN, USA
}

\begin{abstract}
Introduction: Until recently, systemic chemotherapy was the only option for treating bladder cancer and outcomes remained dismal. After a long gap of no progress for 40 years, immunotherapy with checkpoint inhibitors (PDL1 and PD1) has revolutionized the treatment paradigm of bladder cancer, with five approved agents to treat platinum-refractory bladder cancer since the first approval of atezolizumab in May 2016.
\end{abstract}

Methods: This review summarizes the most recent data on approved checkpoint inhibitors currently used in management of advanced bladder cancer. Early- and late-phase trials of the five checkpoint inhibitors (pembrolizumab, nivolumab, atezolizumab, durvalumab, and avelumab) in advanced bladder cancer are reviewed in detail. This review also describes the potential application of PD1/PDL1 inhibitors in adjuvant and neoadjuvant settings and nonmuscle-invasive bladder cancer, as well as with radiation in muscle-invasive bladder cancer treatment. The role of PDL1 and tumor-mutation burden and clinical considerations in choosing a particular immunotherapy are also discussed.

Results: The approved checkpoint inhibitors (PD1 and PDL1 inhibitors) have similar efficacy and safety profiles in metastatic platinum-refractory bladder cancer, but they vary in dose and frequency and cost burden. However, only pembrolizumab has shown superiority over standard chemotherapy in a randomized Phase III setting so far. In addition, in the first-line setting for cisplatin-ineligible patients, both pembrolizumab and atezolizumab are US Food and Drug Administration-approved and well tolerated. There is a lack of consensus on the utility of testing for PDL1 as a predictive biomarker, as patients with no PDL1 expression also derive some clinical benefit. Tumor-mutation burden is another predictive biomarker, but needs further validation.

Conclusion: Immunotherapy has offered a glimmer of hope to patients with bladder cancer. The current landscape is rapidly evolving, with novel immunotherapy-combination trials to improve outcomes further and evaluate predictive biomarkers to help identify patients most likely to benefit from such therapies.

Keywords: pembrolizumab, checkpoint inhibitors, urothelial cancer, tumor-mutation burden, bladder cancer, immunotherapy

\section{Introduction}

Bladder cancer accounts for approximately $4.7 \%$ of all malignancies in the USA. ${ }^{1}$ In 2017, there were an estimated 79,030 bladder cancer cases and 16,870 deaths in the US. ${ }^{1}$ Urothelial carcinoma (UC) of the bladder represents $90 \%$ of cases, while the remaining UC cases occur in the ureter, urethra, and urachus. Around $75 \%$ of bladder cancers are non-muscle-invasive bladder cancer (NMIBC). ${ }^{2}$ Up to $50 \%$ of NMIBC patients who are treated with transurethral resection have disease recurrence, and up to $25 \%$ of these patients progress to muscle-invasive disease after repeated 
recurrences. ${ }^{2}$ Muscle-invasive UC of the bladder accounts for $20 \%-40 \%$ of cases, and the standard of care is radical cystectomy with or without neoadjuvant chemotherapy or concurrent chemoradiation as a bladder-sparing option. ${ }^{3}$ However, even after treatment, up to $50 \%$ patients develop recurrence and most patients die of metastatic disease within 3 years of diagnosis. ${ }^{4}$ De novo metastatic disease is present at diagnosis in $4 \%$ of patients. ${ }^{1}$ Patients with metastatic disease (de novo or recurrent metastatic disease) are incurable, and 5-year relative survival remains dismal.

Prior to the advent of PD1/PDL1 checkpoint inhibitors, systemic chemotherapy with cisplatin-based regimens was the standard of care, leading to median survival of around 1 year. ${ }^{5,6}$ For patients with platinum-refractory disease, the median survival was only 6-9 months..$^{7-10}$ Furthermore, up to $30 \%-50 \%$ of patients with metastatic UC are ineligible to receive cisplatin due to comorbidities, limiting treatment options, and until recently carboplatin-based regimes were the only treatment options, with no substantial improvement in clinical outcomes. ${ }^{11-13}$

After a long void of over four decades, we have made seismic progress in treatment of UC with the approval of several PD1/PDL1 inhibitors in metastatic UC, after the first approval of atezolizumab by the US Food and Drug Administration (FDA) in refractory UC in May 2016. PD1/PDL1 inhibitors are now being studied in a plethora of combinations with other immunotherapeutic and targeted agents to aim further to improve outcomes in various stages of UC. In this review, we assess the role of anti-PD1/PDL1 therapies in the treatment of advanced bladder cancer.

\section{Rationale of immunotherapy use in urothelial carcinoma}

Bladder cancer has been known to be immunoresponsive since original reports by Morales et al on the activity of intravesical instillation of bacillus Calmette-Guérin (BCG) in NMIBC. ${ }^{14}$ $\mathrm{BCG}$ is a live attenuated tuberculosis-related bacteria that stimulates an immunologic reaction inducing a proinflammatory cytokine and direct cell-to-cell cytotoxicity, and was the first immunotherapy approved for NMIBC by the FDA in 1990. ${ }^{14,15}$ Bladder tumors have the third-highest rate of somatic mutations and are highly antigenic. ${ }^{16}$ Additionally, therapeutic agents can be placed in direct contact with the tumor through intravesical administration, allowing a high concentration of the agent to be delivered with limited systemic exposure. Even today, BCG remains a standard of care for patients with NMIBC, and this therapy provides "proof of principle" that $\mathrm{UC}$ can be treated effectively with immunotherapy.
The high rate of tumor mutations in bladder cancer makes it a ripe environment for immunotherapy, and higher mutation rates have been shown to be associated with higher responses to immune-checkpoint blockade in melanoma, lung cancer, and more recently the exploratory subgroup analysis from the IMvigor 210 trial of atezolizumab in UC. ${ }^{17-19}$ It is also possible to monitor treatment response through direct surveillance of the tumor and related cells and biomarkers. The antitumor cellular immunity process is subject to complex modulatory mechanisms in the tumor microenvironment. Inhibitory checkpoints, such as PD1 present on T-cells and its ligands PDL1 and PDL2 regulate T-cell behavior in differentiating self from cancerous cells or pathogens. ${ }^{20}$ The binding of PDL1, expressed on some cancer cells and antigen-presenting cells, to PD1 on activated T cells results in suppression of an immunoresponse. ${ }^{20}$ Therefore, through expression of PDL1, cancer cells avoid immunorecognition by co-opting the PD1 ligands to "cloak" them from immunodetection and destruction by imitating the signaling process of healthy cells. PD1 and PDL1 monoclonal antibodies were developed to inhibit these T-cell-regulatory pathways, promoting T-cell activation against the tumor cells. ${ }^{20}$ Early evidence of the activity of checkpoint inhibition in UC has paved the way for PD1/PDL1 inhibitors to be incorporated in every stage of UC, either alone or in combination with other novel therapies or chemotherapy.

\section{Anti-PDI and PDLI therapies for urothelial cancer}

The PD1 and PDL1 checkpoint inhibitors have emerged as a new treatment option for advanced or metastatic UC. Pembrolizumab and nivolumab are the two PD1 inhibitors and atezolizumab, durvalumab, and avelumab the three PDL1 inhibitors approved by the FDA for refractory metastatic UC (Table 1). Two of these PD1/PDL1 inhibitors have received orphan designation from the FDA, and all five of the PD1/PDL1 inhibitors benefited from the FDA's accelerated approval process. In the following sections, we discuss the key clinical trials providing evidence of safety and efficacy of FDA-approved PD1/PDL1 therapies for UC.

\section{Pembrolizumab}

Pembrolizumab is a humanized monoclonal $\operatorname{IgG}_{4}$ PD1 antibody blocking the signaling of both PDL1 and PDL2 ligands. This drug has shown antitumor activity in humans with a reasonable safety profile and been approved in more than 60 countries. Pembrolizumab was the first checkpoint inhibitor approved by the FDA, and it is now approved to 


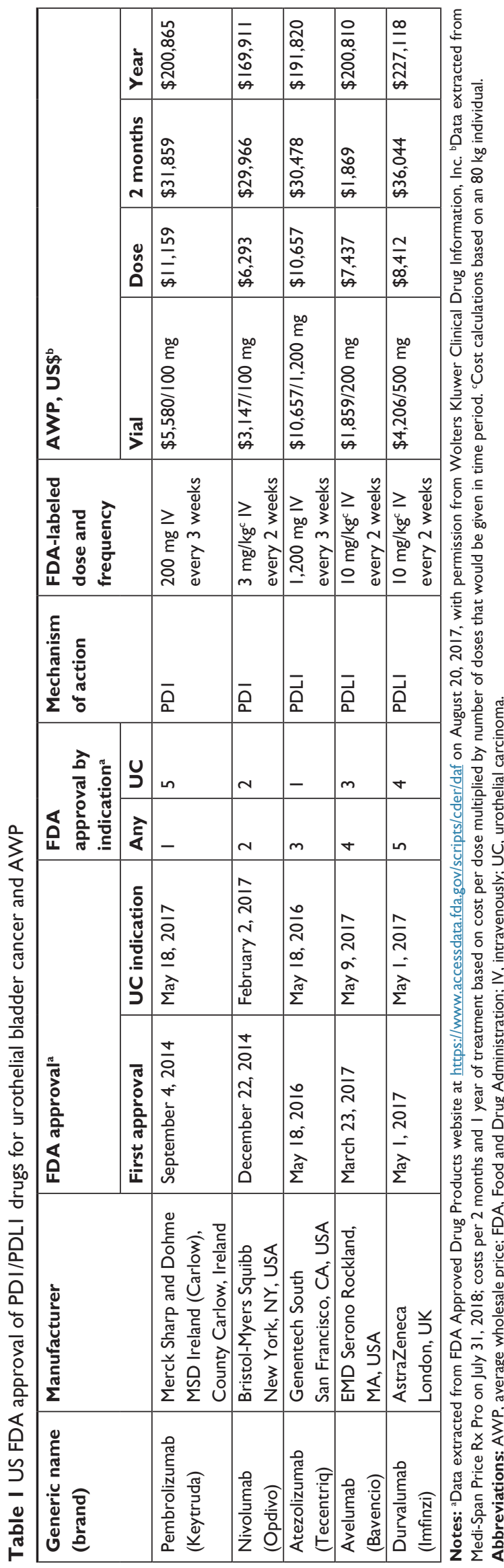

treat at least six conditions as either a single agent or in combination with ipilimumab. ${ }^{21}$ In metastatic or locally advanced UC, pembrolizumab is approved for patients who are not eligible for cisplatin-containing chemotherapy and those who have disease progression during or following platinum-containing chemotherapy. ${ }^{21}$ FDA approval for the first of these indications was based on an accelerated approval process using tumor-response rate and duration of response (DoR), and confirmatory trials have been requested by the FDA. ${ }^{21}$ The approval of pembrolizumab was based upon five studies using randomized, open-label, activecontrol clinical trials (KEYNOTE-00222, KEYNOTE-006 ${ }^{23}$, KEYNOTE-010 $0^{24}$, KEYNOTE-021 $1^{25}$, AND KEYNOTE$045^{26}$ ) with 542 patients with $\mathrm{UC}$ and three nonrandomized, open-label trials (KEYNOTE-012 ${ }^{27}$, KEYNOTE-052 ${ }^{28}$, AND KEYNOTE- $087^{29}$ ) with 370 UC patients. In the following section, we summarize the key trials leading to FDA approval of pembrolizumab in advanced UC, both in patients who are platinum-ineligible and those who have progressed during or after platinum-containing chemotherapy.

\section{Pembrolizumab in refractory metastatic UC for} progression on platinum-containing chemotherapy The KEYNOTE- $012^{27}$ study was a nonrandomized, multicohort, open-label, Phase 1B trial that enrolled patients with a confirmed diagnosis of locally advanced or metastatic UC. No requirement for prior treatment was mandated. Patients were screened for PDL1 biomarker-expression status in tumor cells, and only those with $>1 \%$ expression were included in the study. Initially, 115 patients were screened, and 61 (53\%) were positive for PDL1 expression. Of the 61 PDL1-positive patients, 33 were enrolled in the study.

The primary end-point measures were safety, tolerability, and objective response rate (ORR) as per RECIST (responseevaluation criteria in solid tumors) version 1.1. by central review. Secondary end points were OR based on investigator assessment, progression-free survival (PFS), overall survival (OS), and DoR. A total of 33 patients received at least one dose of pembrolizumab. Subject characteristics included $91 \%$ of patients with urothelial histology. Three-quarters of the patients $(76 \% ; 25$ of 33$)$ had received at least one treatment for locally advanced or metastatic disease, and the remaining $24 \%$ had received platinum-based therapy in the adjuvant or neoadjuvant setting before disease progression. The median time on pembrolizumab was 71 days. Adverse events (AEs) were reported by $61 \%$ (20 of 33) of patients, while $39 \%$ had no treatment-related AEs. The most common AEs reported were fatigue (18\%) and peripheral edema (12\%). 
Five patients had grade 3 treatment-related AEs, and two of those discontinued therapy. Six (18\%) patients had interruptions in treatment due to AEs. Eight immunorelated events, including myositis, uveitis, colitis, pruritic rash, maculopapular rash, and rhabdomyolysis, were experienced by six patients $(18 \%)$. No deaths on the study were considered treatment-related.

The median duration of follow-up was 13 months, and 27 patients were assessed for response. Seven of 27 patients (26\%) achieved an OR, three had a complete response, and four had a partial response. The median time to response was 2 months, and the median DoR was 10 months. Sixteen of $25(64 \%)$ patients with response data had reductions in tumor size, with eight patients having $>30 \%$ reduction. There were no cases of pseudoprogression. Median PFS was 2 months. The PFS rate at 12 months was 15\%. Median OS was 13 months, and OS at 12 months was 50\%. The median OS of 13 months was considered a significant improvement over the typical median survival time of 6-9 months with chemotherapy in the second-line setting. ${ }^{30}$

A post hoc exploratory analysis was conducted to correlate baseline PDL1 expression with clinical response. For screening, a prototype assay (QualTek Molecular Laboratories; Goleta, CA, USA) was utilized to determine PDL1 positivity. A separate clinical trial assay procedure was used to determine presence of PDL1 staining in tumor cells and in tumor cells plus inflammatory cells "combined score". In this assay, four patients were considered PDL1-negative who had been PDL1-positive in the screening assay. The second method used commercially available reagents from the Dako EnVision Flex + HRP polymer kit in combination with the anti-PDL1 clone 22C3 antibody, which was also used in the prototype assay; however, the assays have different staining methodologies and definitions of PDL1 positivity. Only 25 patients had PDL1-expression data with the clinical assay and had response data available. Based on tumor cells alone, 14 of 25 (56\%) patients had positive PDL1-biomarker status. When both tumor cells and inflammatory cells were considered, 21 of 25 (84\%) patients were positive for PDL1 staining. Use of inflammatory cells along with tumor cells helped differentiate responders to therapy, with a 24\% ORR in PDL1-positive vs 0 in PDL1-negative. When only tumor cells were used to determine PDL1 positivity, a $14 \%$ ORR was observed compared to $27 \%$ in the PDL1-negative subgroup.

The KEYNOTE-012 study demonstrated preliminary activity of pembrolizumab with a manageable side-effect profile in advanced UC, with the majority of subjects receiving at least one line of prior systemic chemotherapy. The KEYNOTE- $045^{26}$ study was a randomized, open-label, active-controlled Phase III clinical trial comparing the efficacy of pembrolizumab with investigator-choice chemotherapy among patients who had previously been treated for metastatic UC. Patients were also included if they had experienced recurrence within 12 months of platinum-based adjuvant or neoadjuvant therapy. Patients in this trial were given either a dose of $200 \mathrm{mg}$ pembrolizumab intravenously every 3 weeks (270 patients) or standard-of-care single-agent chemotherapy with paclitaxel or docetaxel or vinflunine (272 patients). No PDL1-expression requirement was mandated for trial inclusion. The coprimary end points were OS and PFS. Secondary efficacy end points were ORR and DoR. End points were assessed in the total population and in those with a tumor-PDL1 combined positive score (CPS) $\geq 10 \%$.

Median age was 66 years, 74\% were male, $87 \%$ had visceral metastases, $15 \%$ had disease progression after platinum-containing chemotherapy, and 76\% had received prior cisplatin. Median follow-up across both groups was 9.0 months. Approximately $28.5 \%$ and $33.8 \%$ of patients in the pembrolizumab and chemotherapy groups, respectively, had a tumor-PDL1 CPS $\geq 10 \%$ of tumor and immune cells (ICs; Dako PDL1 IHC 22C3 PharmDx assay).

The results showed an improvement in OS (10.3 vs 7.4 months) in favor of pembrolizumab (HR $0.73,95 \% \mathrm{CI}$ $0.59-0.91 ; P=0.002)$. OS in patients with a combined PDL1 score $\geq 10 \%$ was 8 months in the pembrolizumab group vs 5.2 months in the chemotherapy group $(P=0.005)$. The ORR was $21.1 \%$ vs $11.4 \%$ (measured by RECIST 1.1 ) for pembrolizumab vs single-agent chemotherapy in the total population $(P=0.001)$. PFS was not statistically different between the two study groups, nor was the comparison of those with $\geq 10 \%$ PDL1 combined score.

Fewer serious AEs (grade 3-5) were reported for patients using pembrolizumab than for patients on chemotherapy $(15.0 \%$ vs $49.4 \%) .{ }^{26}$ Treatment-related AEs occurred in $60.9 \%$ of patients in the pembrolizumab group compared to $90.2 \%$ of those in the chemotherapy group. The most common treatment-related AEs in the pembrolizumab group were pruritus (19.5\%), fatigue (13.9\%), and nausea (10.9\%). Immunorelated AEs were observed in $16.9 \%$ of patients in the pembrolizumab group, with hypothyroidism being most common (6.4\%). This study demonstrated that pembrolizumab improves OS with reduced AEs compared to investigator-choice single-agent chemotherapy in those who had progressed after platinum-based chemotherapy for metastatic disease or within 12 months after platinum-based 
adjuvant or neoadjuvant chemotherapy, and represents the first and only randomized Phase III study demonstrating an OS benefit of a checkpoint inhibitor in the second-line setting and supported FDA approval of pembrolizumab.

\section{Pembrolizumab in first-line cisplatin-ineligible metastatic UC}

Up to $30 \%-50 \%$ of metastatic UC patients are ineligible to receive cisplatin and development of safe and effective novel therapies to improve outcomes, and limiting toxicity in this "cisplatin-ineligible" patient population is an unmet need. ${ }^{11-13}$ The KEYNOTE-052 28 study was a Phase II single-arm clinical trial assessing the safety of pembrolizumab as firstline therapy for patients with locally advanced or metastatic UC who were not eligible for cisplatin-containing chemotherapy. As in the KEYNOTE-045 study, patients were not required to have PDL1 expression for inclusion in the trial. The dose of pembrolizumab was $200 \mathrm{mg}$ every 3 weeks and continued until unacceptable toxicity, radiographic evidence of progression, or clinical evidence of disease progression. The primary end point was ORR. The total enrollment was 370 patients, and all patients were included in the safety analysis. Updated analysis from Keynote-052 showed ORR to be $29 \%$, including $7 \%$ complete-response and $22 \%$ PR rates. In the training set of the first 100 patients in this study, patients with a CPS $<10 \%$ had an ORR of $17 \%$, and ORR was 37\% for CPS-high patients, and in the validation set the response was even stronger, with ORR 51\% for CPS-high patients, and 23\% for CPS-low patients. Pembrolizumab was well tolerated, with $19 \%$ patients reporting three or fewer AEs, including fatigue $(2 \%)$ and colitis $(2 \%) .{ }^{31}$ The median time to response was 2 months and median duration of treatment 3 months. More than three-quarters (78\%) of the responders had a response lasting $>6$ months. The median PFS was 2 months and the 6-month PFS and OS rates 30\% and $67 \%$, respectively.

ORR was also assessed by PDL1 expression in both tumor cells and ICs using the same combined score method in the KEYNOTE-045 and -012 studies (Dako PDL1 IHC 22C3 PharmDx assay). Receiver-operating characteristic analysis was conducted to determine PDL1-expression cutoffs ( $1 \%$, $5 \%$, and $10 \%$ ) predictive of response to pembrolizumab. In the training set, combined positive PDL1 score $\geq 10 \%$ was considered optimal. In the validation cohort, an increased response rate was observed with this cutoff; however, it was not $100 \%$ predictive, as responses were still observed at the lower cutoff values, necessitating future research before clinical use of this biomarker.
Treatment-related AEs were experienced by $62 \%$ of patients, with $16 \%$ experiencing a grade $\geq 3$ AE. Pembrolizumab was discontinued due to AEs in $5 \%$ of patients. Eighteen patients (5\%) died during the study. One death was considered treatment-related (myositis; grade 3 thyroiditis, hepatitis, and pneumonia; grade four myocarditis). Immunorelated AEs were observed in $17 \%$ of patients, most commonly hypothyroidism (6\%), hyperthyroidism $(2 \%)$, colitis $(2 \%)$, and pneumonitis $(2 \%)$. The most common grade $\geq 3$ treatment-related AEs were fatigue, alkaline phosphatase increase, colitis, and muscle weakness.

Across these three studies and two populations of patients (first- and second-line treatment) pembrolizumab ORR was remarkably similar: $26 \%, 21 \%$, and $24 \%$ for KEYNOTE$012,-052$, and -045 , respectively. (Table 2). The AE rate was also consistent across trials, with $61 \%-62 \%$ of patients experiencing treatment-related AEs and $17 \%-18 \%$ of patients reporting immunorelated AEs. Tumor and immune-cell expression of PDL1 correlated with response to pembrolizumab, but lacked specificity, limiting its application as a companion diagnostic.

While pembrolizumab is the only checkpoint inhibitor that has demonstrated superiority over standard chemotherapy in a randomized Phase III trial in refractory setting so far, there are four other checkpoint inhibitors currently approved by the FDA for refractory metastatic UC based on single-arm Phase II trials. In addition, atezolizumab is also approved in the first-line treatment of metastatic cisplatin-ineligible UC. Clinical trial data pertaining to these checkpoint inhibitors is reviewed in the following sections.

\section{Atezolizumab}

Atezolizumab is a humanized $\mathrm{IgG}_{1}$ monoclonal antibody to PDL1, and was the first PD1/PDL1 inhibitor approved by the FDA for treatment of advanced $\mathrm{UC}$, based on results from the IMvigor 210 clinical study. ${ }^{19,32}$ The approved indications for atezolizumab in advanced UC are similar to pembrolizumab: disease progression during or following platinum-containing chemotherapy, and first-line treatment for cisplatin-ineligible patients. ${ }^{33}$ Approval of atezolizumab by the FDA was based on tumor-response rate and durability of response. The FDA noted in the approved labeling that "continued approval for this indication may be contingent upon verification and description of clinical benefit in confirmatory trials". ${ }^{33}$

The IMvigor 210 clinical study was a Phase II, multicenter, open-label trial of atezolizumab with two cohorts and provided the evidence for the FDA-approval decisions. ${ }^{19,32}$ Cohort 1 included advanced UC patients who were ineligible 


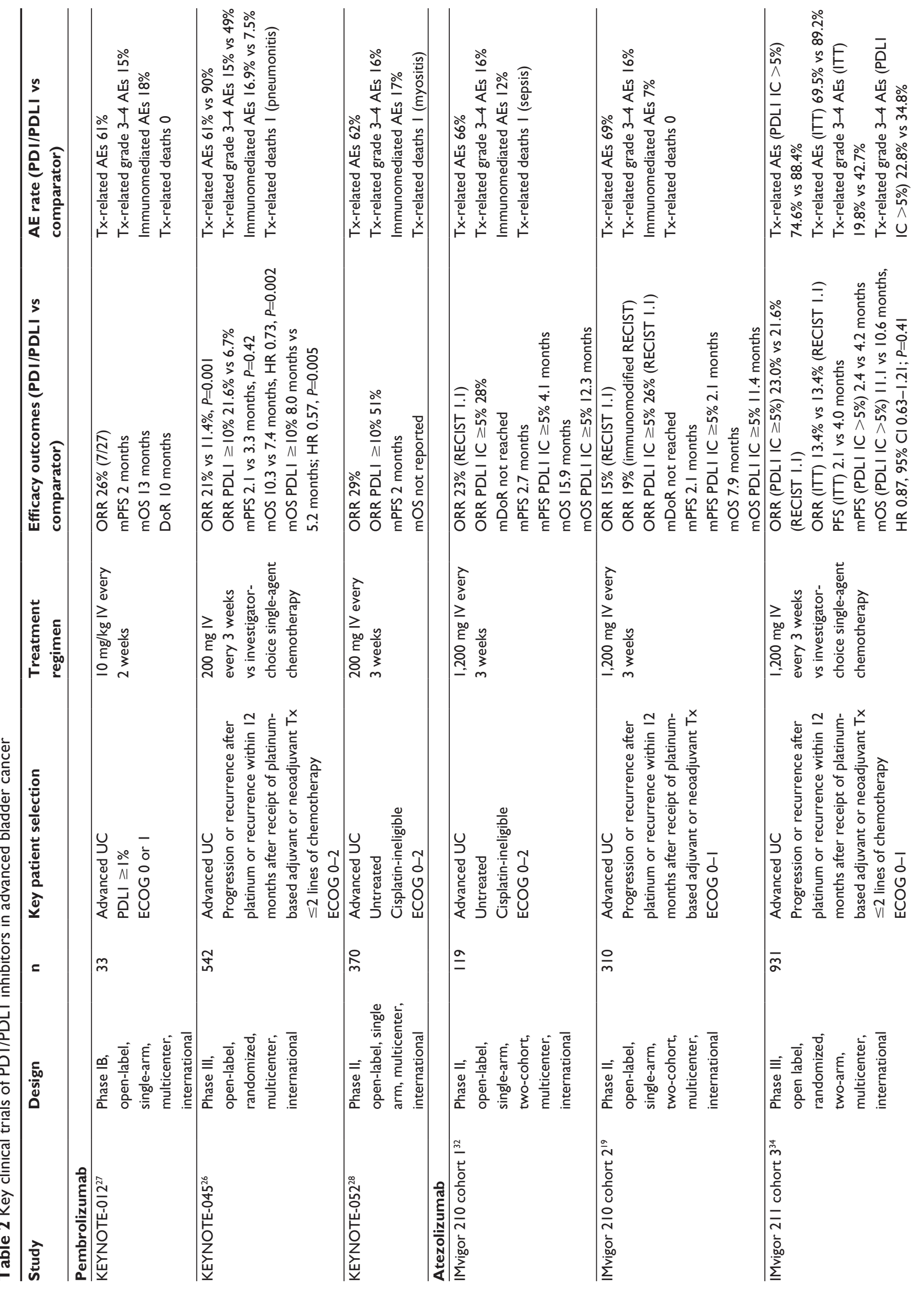




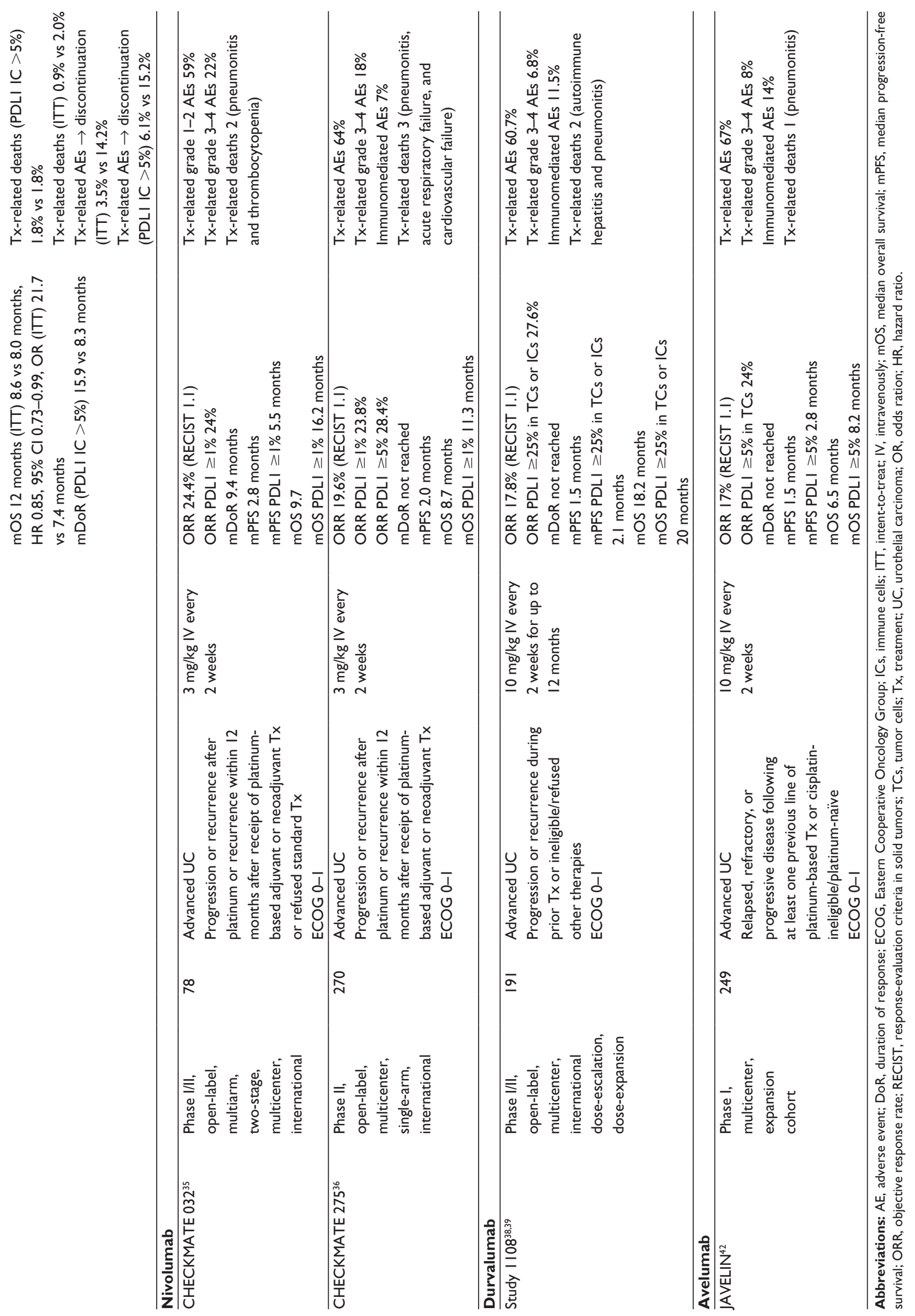


for first-line treatment with cisplatin. ${ }^{32}$ Cohort 2 included patients with disease progression or recurrence after platinum therapy (second-line treatment). ${ }^{19}$ In both cohorts, patients were administered atezolizumab 1,200 mg every 3 weeks until unacceptable or either radiographic or clinical progression. PDL1 expression on tumor-infiltrating ICs was prospectively assessed using the Ventana PDL1 IHC (SP142) assay. Patients were classified by percentage PDL1 expression on ICs ( $\mathrm{IC}_{0}<1 \%$ of ICs, $\mathrm{IC}_{1} 1 \%-5 \%, \mathrm{IC}_{2 / 3} \geq 5 \%$ ). The primary outcome measure was ORR, and secondary outcomes included DoR, PFS, and OS in both cohorts. Disease assessments occurred every 9 weeks.

In cohort 1 of IMvigor 210, 119 patients received atezolizumab as first-line treatment. The ORR was $23 \%$, and $9 \%$ of all patients achieved a complete response. ORs were observed in all PDL1-expression cohorts, and ranged from $21 \%$ in $\mathrm{IC}_{0}$ to $28 \%$ in $\mathrm{IC}_{2 / 3}$. The median DoR had not been reached at trial publication (range 3.7-21.0+ months). Median PFS was 2.7 months and median OS 15.9 months. In exploratory analysis, patients in the highest quartile of tumor-mutation load per megabase demonstrated improved survival. Unlike as seen with pembrolizumab in this setting, PDL1 expression did not correlate with clinical outcomes with atezolizumab. The AE rate was similar to other PD1/PDL1 inhibitors, with treatment-related AEs observed in $66 \%$ of patients and $15 \%$ experiencing a grade 3-4 AE. Immunomediated AEs were noted in $12 \%$ of patients. One death (sepsis) was considered treatment-related. The favorable results from cohort 1 of IMvigor 210 supported accelerated FDA approval for this indication, and a confirmatory trial is being conducted (IMvigor 130, NCT02807636).

In cohort 2 of IMvigor 210, 310 patients received atezolizumab and were evaluable for safety and efficacy assessment. ${ }^{19}$ The ORR was $15 \%$ in the total population compared to $26 \%$ in the PDL1 $\mathrm{IC}_{2 / 3}$ subgroup by RECIST 1.1 with independent review. The median DoR was not reached, but $84 \%$ of patients maintained a response after a median 11.7 months of follow-up. Median PFS was 2.1 months, which was the same in the PDL1 $\mathrm{IC}_{2 / 3}$ subgroup. Median OS was 7.9 months in the total population and 11.4 months in the PDL1 $\mathrm{IC}_{2 / 3}$ subgroup. The study protocol allowed treatment beyond progression, which occurred in 120 patients (39\%), and $17 \%$ of these patients had a reduction in tumor volume of at least $30 \%$ following RECIST 1.1 progression. Treatment-related AEs of any grade were observed in $69 \%$ of patients, with grade $3-4$ events occurring in $16 \%$. Fatigue was the most common treatmentrelated grade 3-4 AE. Immunomediated AEs occurred in 7\% of patients, with no treatment-related deaths observed.
Exploratory analyses of PDL1 IC prevalence was significantly increased in Cancer Genome Atlas basal subtype compared to luminal subtype ( $60 \%$ vs $23 \%$ ), but did not correlate with response rates. Mutational load was also significantly greater in those with an OR compared to nonresponders (12.4 vs 6.4 mutations per megabase). Overall, this study demonstrated preliminary evidence of efficacy in advanced UC after platinum-based therapy. PDL1 IC expression correlated with response rates; however, responses were observed in all subgroups. Tumor-mutation burden was demonstrated to be a potential biomarker for response to PD1/PDL1 inhibition.

The IMvigor study was a nonrandomized, multicohort, open-label, Phase III trial that enrolled patients with confirmed diagnoses of metastatic UC who had progressed after platinum-based chemotherapy. Based on the favorable results of cohort 2 in IMvigor 210, a pivotal Phase III confirmatory study (IMvigor 211, NCT2302807) was conducted comparing atezolizumab to chemotherapy (investigator's choice) in the second-line setting after platinum chemotherapy for advanced urothelial bladder cancer. IMvigor $211^{34}$ was the first Phase III randomized trial reporting results for PDL1antibody status in patients with metabolic UC. IMvigor 211 randomly assigned 931 patients from 198 sites into two groups: atezolizumab $(n=467)$ and investigator's choice of chemotherapy $(\mathrm{n}=464)$. All patients were screened and grouped by PDL1 biomarker-expression status (ie, $\mathrm{IC}_{0}-\mathrm{IC}_{2 / 3}$ ) using the Ventana SP142 PDL1 assay. Patients, investigators, and the sponsor were blinded to the PDL1 status of patients. There were 234 patients with a PDL1 status of $\mathrm{IC}_{2 / 3}$, of whom 116 were randomized to the atezolizumab group and 118 to the chemotherapy group. Also, the tumor-mutation burden was analyzed using the Foundation Medicine algorithm for extrapolating genomic alterations detected to the whole exome or genome, and subjects were categorized as high (at or above the median) or low (less than the median).

Primary efficacy analysis was conducted on the $234 \mathrm{IC}_{2 / 3}$ patients for the primary end point (ie, OS rate), and did not show a statistically significant difference between the atezolizumab and chemotherapy groups (median 11.1 [95\% CI 8.6-15.5] months vs 10.6 [95\% CI 8.4-12.2] months, respectively; stratified HR 0.87 [95\% CI $0.63-1.21, P=0.41]$ ). The confirmed ORR in the $\mathrm{IC}_{2 / 3}$ population was similar between the two treatment groups, with $23 \%$ (26 of 113) in the atezolizumab-treated group and $22 \%$ ( 25 of 116) in the chemotherapy-treated group. The DoR was numerically longer in the atezolizumab group than in the chemotherapy group (15.9 months vs 8.3 months). Exploratory analysis 
showed that year-1 milestone survival rates were notably better with the atezolizumab group (467 patients) than the chemotherapy group (464 patients) in the intent-to-treat population (39.2\% vs $32.4 \%$, respectively).

Among the $\mathrm{IC}_{2 / 3}$ patients, treatment-related AEs led to discontinuation of therapy for $6 \%$ (seven of 114) of the atezolizumab group and 15\% (17 of 112) of the chemotherapy group. In the broader intent-to-treat population, AEs led to discontinuation of therapy for 3\% (16 of 459) of the atezolizumab group and 14\% (63 of 443) of the chemotherapy group. AEs of any grade were reported as treatment-related for $75 \%$ ( 85 of 114) of the atezolizumab group and $88 \%$ ( 99 of 112) of the chemotherapy group. The most common AEs, reported in $>10 \%$ of patients in both the $\mathrm{IC}_{2}$ and $\mathrm{IC}_{3}$ groups (atezolizumab vs chemotherapy-treated populations) were fatigue ( $16 \%$ vs $24 \%$ ), pruritus ( $12 \%$ vs $3 \%$ ), esthesia ( $12 \%$ vs $21 \%)$, rash ( $11 \%$ vs $6 \%$ ), pyrexia ( $11 \%$ vs $4 \%$ ), decreased appetite ( $10 \%$ vs $18 \%)$, and diarrhea ( $10 \%$ vs $13 \%)$.

IMvigor 211 demonstrated that atezolizumab is well tolerated (ie, similar or fewer AEs) with a durable remission rate compared with chemotherapy in the treatment of patients with metastatic UC who had previously been treated with platinum-containing therapy. ${ }^{34}$ However, atezolizumab failed to demonstrate an OS benefit compared to chemotherapy in the PDL1-expressing $\mathrm{IC}_{2 / 3}$ population, which is contrary to the Phase III results with pembrolizumab. Differences in PDL1 assays may at least partially explain these results.

\section{Nivolumab}

Nivolumab is a fully human $\mathrm{IgG}_{4}$ PD1-inhibitor antibody. Nivolumab was first approved by the FDA in December 2014 for the treatment of patients with unresectable or metastatic melanoma and disease progression following ipilimumab (Yervoy). At least eight additional indications have been approved for nivolumab. Nivolumab was granted accelerated approved by the FDA in February 2017 for treating patients with locally advanced or metastatic UC with disease progression during or following platinum-containing chemotherapy or disease progression within 12 months of neoadjuvant or adjuvant treatment with platinum-containing chemotherapy.

Nivolumab received the FDA breakthrough-therapy designation based on the findings of CHECKMATE 032 study. ${ }^{35}$ This was a multiarm, multicenter, open-label, Phase I/II study in recurrent metastatic UC after platinumbased chemotherapy. The primary end point was ORR and secondary end points safety, DoR, PFS, and OS. PDL1-tumor expression was assessed retrospectively using the Dako
PDL1 IHC 28-8 PharmDx kit. PDL1 tumor expression was categorized positive if $\geq 1 \%$ staining. Patients received nivolumab $3 \mathrm{mg} / \mathrm{kg}$ intravenously every 2 weeks.

The ORR was $24.4 \%$ in the total population and was similar (24\%) in the PDL1 $\geq 1 \%$ subgroup. The median DoR was 9.4 months. Median PFS was 2.8 months in the total population and 5.5 months in the PDL1 $\geq 1 \%$ subgroup. Similarly, median OS was 9.7 months in the total population and 16.2 months in the PDL1 $\geq 1 \%$ subgroup. Grade 3-4 treatment-related AEs occurred in $22 \%$ of patients. Two patients died due to treatment-related AEs (grade 4 pneumonitis and grade 4 thrombocytopenia). This study provided preliminary evidence of the safety and efficacy of nivolumab in the second-line setting after platinum-based chemotherapy for advanced UC. PDL1 expression using only tumor cells was not associated with ORR; however, differences were observed in PFS and OS.

The CHECKMATE $275^{36}$ study was conducted to characterize further the efficacy and safety of nivolumab in advanced UC after platinum-based chemotherapy. This was a single-arm, multicenter, Phase II study. Tumor-cell PDL1 expression was assessed by the same assay (Dako) as in CHECKMATE 032; however, cutoffs for positivity included $\geq 1 \%$ and $\geq 5 \%$. The primary end point was ORR in all patients and in PDL1 tumor expression $\geq 5 \%$. A total of 270 patients received nivolumab on the study. ORR in all patients was $19.6 \%$, and was $23.8 \%$ and $28.4 \%$ in PDL1 expression $\geq 1 \%$ and $\geq 5 \%$, respectively. Median DoR was not reached, but $77 \%$ were still responding at publication. Median PFS was 2 months. Median OS was 8.7 months, and was 11.3 months in those with tumor expression of PDL1 $\geq 1 \%$ ( $\geq 5 \%$ not reported). Treatment-related AEs were observed in $64 \%$ of patients, most commonly fatigue. Grade 3-4 treatment-related AEs were observed in 18\%, most commonly fatigue and diarrhea. The most common immunomediated AEs were skin (17\%) and endocrine (14\%). Three deaths were considered treatment-related (pneumonitis, acute respiratory failure, and cardiovascular failure).

Exploratory analyses demonstrated IFN $\gamma$ gene expression was associated with increased ORR and PDL1 tumor-cell expression. Similarly to the IMvigor 210 cohort 2 study, Cancer Genome Atlas basal subtype was associated with an increased proportion of responders and was also shown to have enrichment for IFN $\gamma$ signature. The CHECKMATE $275^{36}$ study provided single-arm evidence of the activity and safety of nivolumab in the second-line setting. PDL1 tumor-cell expression did not correlate with ORR, and responses were observed at all expression levels. Further study is warranted 
to elucidate the predictive value of an IFN $\gamma$ signature and basal subtype with response to PD1/PDL1 inhibition.

\section{Durvalumab}

Durvalumab is a human $\operatorname{IgG}_{1 \mathrm{~K}}$ monoclonal PDL1-antibody inhibitor and was granted accelerated approval by the FDA in May 2017 for locally advanced or metastatic UC in the second-line treatment setting. ${ }^{37}$ The efficacy of durvalumab was studied in a UC dose-expansion cohort in a large Phase I/II, multicenter, multicohort, open-label clinical trial (Study 1108). ${ }^{38,39}$ In this cohort, 191 patients with locally advanced or metastatic UC were enrolled. The patients had disease that had progressed on prior therapy $(99.5 \%)$ or were treatment-naïve. The majority of patients (95.3\%) had disease that had progressed during or after platinumbased chemotherapy or within 12 months of adjuvant or neoadjuvant therapy. PDL1 expression was assessed prior to treatment. The Ventana SP263 anti-PDL1 antibody assay was utilized. The first 20 patients were enrolled regardless of PDL1 expression. The next 43 patients were required to have $\geq 5 \%$ PDL1 expression on tumor cells. The protocol was amended to remove this requirement after an interim analysis demonstrated similar ORR by PDL1 expression. This study used a $\geq 25 \%$ cutoff for IC or tumor-cell expression for biomarker development. Durvalumab was administered every 2 weeks at $10 \mathrm{mg} / \mathrm{kg}$ intravenously for up to 12 months or until unacceptable toxicity or disease progression. Tumor assessments were done at weeks 6,12 , and 16 , then every 8 weeks for the first year and every 12 weeks thereafter. The primary efficacy outcome measures were safety and ORR.

An ORR of $17.8 \%$ by RECIST 1.1 was observed, with complete responses occurring in $3.7 \%$. When subjects in the cohort were assessed by PDL1-expression status, the high group ( $\geq 25 \%$ PDL1 tumor cells or ICs) had an ORR of $27.6 \%$ and the low/negative group had an ORR of $5.1 \%$. Median DoR had not yet been reached for study subjects at the time of data reporting. Half the responding subjects had an ongoing response at 6 months. Median PFS and OS were 1.5 and 18.2 months in the total population and 2.1 and 20 months in the PDL1 $\geq 25 \%$ tumor-cell or IC subgroup, respectively. Treatment-related AEs were observed in $60.7 \%$ of patients, with fatigue being most common (19.4\%). Grade 3-4 treatment-related AEs occurred in 6.8\% of patients. Immunomediated AEs were reported in 20.5\% of patients, and two treatment-related AEs resulted in death (autoimmune hepatitis and pneumonitis).

Overall, durvalumab demonstrated preliminary safety and efficacy in this dose-expansion cohort. Similarly to the other PD1/PDL1 studies, despite the enrichment of responses in those with high PDL1 expression in tumor cells and ICs, responses were still observed in those with low or no expression. Based on the accelerated approval, confirmatory trials are underway, including a Phase III trial (DANUBE, NCT02516241) in the first-line setting comparing durvalumab with and without tremelimumab (anti-CTLA4) to standard-of-care chemotherapy.

\section{Avelumab}

Avelumab is a human $\operatorname{IgG}_{1}$ monoclonal PDL1-antibody inhibitor. It was approved by the FDA in May 2017 for treatment of patients with UC in the second-line treatment setting. Like the other PD1/PDL1 inhibitors, this FDA approval was an accelerated approval, and confirmatory trials are mandated. ${ }^{40}$ The JAVELIN Solid Tumor trial was the basis for demonstrating efficacy and safety to support the accelerated FDA approval. ${ }^{41,42}$ This was an open-label, single-arm, multicenter study. Patients were included if they had progressed after platinum-based chemotherapy or if they were cisplatin-ineligible or platinum-naïve. PDL1 tumor expression was assessed by IHC with Dako PDL1 IHC73-10 PharmDx assay (anti-PDL1 clone 73-10 antibody) and a cutoff of $\geq 5 \%$ tumor-cell PDL1 expression chosen for analysis, but patients were included regardless of their PDL1 status. Patients were given avelumab at a dose of $10 \mathrm{mg} / \mathrm{kg}$ intravenously every 2 weeks until radiographic or clinical progression or unacceptable toxicity. Primary end points were safety in the dose-escalation portion of the study and ORR as per RECIST 1.1 by independent review in dose expansion.

A total of 249 patients were enrolled and received avelumab. Only $2.8 \%$ of patients had not received prior platinum-based chemotherapy and in total 5\% were considered platinum-ineligible at enrollment. Prior drug therapy was administered in $98 \%$ of participants. Efficacy assessment was conducted in 161 patients who received prior platinum-based therapy and had 6 months of follow-up. ORR was $17 \%$, with $6 \%$ complete-response rate in 161 patients. ORR in PDL1 $\geq 5 \%$ in tumor cells was $24 \%$. Median DoR was not reached; however, $96 \%$ were still responding after 24 weeks. Median PFS was 6.3 weeks ( 1.5 months), and disease assessment occurred every 6 weeks. Median PFS in PDL1 $\geq 5 \%$ was 2.8 months. OS was 6.5 and 8.2 months in the total population and the PDL1 $\geq 5 \%$ subgroup. Treatment-related AEs occurred in $67 \%$ of patients, with $8 \%$ grade 3-4. Most common treatment-related AEs were infusion-related reactions ( $29 \%$, all $\leq$ grade 2$)$ and fatigue (16\%, with $2 \%$ grade 3 ). Immunomediated AEs were observed in $14 \%$ of patients, and were commonly rash (10\%) 
and hypothyroidism (4\%). One treatment-related death was attributed to pneumonitis.

The JAVELIN study demonstrated preliminary safety and efficacy for avelumab after platinum-based therapy. PDL1 tumor expression was enriched for responses to avelumab; however, as with other studies, responses were still observed in all subgroups. In exploratory analysis, tumormutation burden was assessed in 29 patients, and although statistical significance was not achieved $(P=0.076)$, a trend was observed for increased tumor-mutation burden by best overall response. In future, tumor-mutation burden coupled with PDL1 expression may improve the predictive estimation of response to avelumab and other PD1/PDL1 inhibitors.

\section{PDI/PDLI inhibitors in clinical practice \\ First-line treatment of cisplatin-ineligible metastatic UC}

In the first-line treatment setting for advanced UC in patients who are cisplatin-ineligible, only pembrolizumab and atezolizumab are FDA-approved, based on results from the KEYNOTE-052 and IMvigor 210 cohort 1 trials, respectively. ${ }^{28,32}$ ORRs in these trials were $29 \%$ and $23 \%$, respectively, with similar AE profile. Notably, in patients with $>10 \%$ PDL1 CPS score, ORR was $51 \%$ with pembrolizumab. According to the current National Comprehensive Cancer Network guidelines, both agents carry a category-2A recommendation for first-line treatment of cisplatin-ineligible advanced or metastatic UC. ${ }^{3}$ Both agents are dosed every 3 weeks at a fixed dose, resulting in similar costs and patient convenience. Ongoing randomized clinical trials will provide information on whether checkpoint inhibitors and checkpoint inhibitor-chemotherapy combinations will result in higher efficacy compared to standard chemotherapy in the first-line cisplatin-ineligible setting.

New findings from early reviews by the respective Data Monitoring Committees (DMC) of two ongoing clinical trials (KEYNOTE-361 and IMVIGOR-130) found that patients with low PD-L1 in the monotherapy arms of both trials had decreased survival compared to patients who received cisplatin- or carboplatin-based chemotherapy. The revised labeling in the FDA Alert (June 2018) reported that pembrolizumab (Keytruda) is now indicated for the treatment of patients with locally advanced or metastatic urothelial carcinoma who are not eligible for cisplatin-containing therapy and whose tumors express PD-L1 (Combined Positive Score (CPS $\geq 10$ ), or in patients who are not eligible for any platinum-containing chemotehrapy regardless of PD-L1 status. The FDA Alert (June 2018) reported that atezolizumab
(Tecentriq) is now indicated for the treatment of patients with locally advanced or metastatic urothelial carcinoma who: 1) are not eligible for cisplatin-containing therapy, and whose tumors express PD-L1 (PD-L1 stained tumor-infiltrating ICs covering $\geq 5 \%$ of the tumor area), or 2 ) are not eligible for any platinum-containing therapy regardless of level of tumor PD-L1 expression. The FDA Alert did not require any change in the adverse event profile of either pembrolizumab or atezolizumab.

\section{Second-line treatment of platinum- refractory metastatic UC}

In the second-line setting after platinum-based chemotherapy or within 12 months after platinum-based adjuvant or neoadjuvant treatment, all five PD1/PDL1 inhibitors discussed herein are FDA-approved, and any of these inhibitors may be used. However, only pembrolizumab carries a National Comprehensive Cancer Network category 1 recommendation for this indication, based on the superiority of pembrolizumab over standard chemotherapy in the Phase III KEYNOTE-045 trial. ${ }^{26}$ The dosing frequency of an individual agent may impact decision-making for providers and patients.

\section{Safety}

The safety profile appears to be similar across the PD1/PDL1 inhibitors (Table 2). In all studies presented, eleven deaths were attributed to PD1/PDL1 inhibition in 1,880 patients $(<0.5 \%)$. Importantly, of these deaths, five were attributed directly to pneumonitis and occurred in those treated with PD1 and PDL1 inhibitors. Future research is needed to identify the role of potential risk factors, such as smoking and radiation. Immunomediated AEs of any grade occurred in $7 \%-18 \%$ of treated patients, and despite the low treatmentrelated death rate, care should be taken with PD1/PDL1 inhibition for early identification of immunomediated AEs and prompt and appropriate management. ${ }^{43-45}$ Patient education and community outreach are crucial to generate awareness regarding immunorelated $\mathrm{AE}$ management.

\section{Pseudoprogression and atypical response kinetics}

In the trials discussed, only KEYNOTE-012 assessed pseudoprogression, and no cases were identified. Median time to response across all trials was approximately 2 months. However, IMvigor 210 cohort 2 allowed treatment beyond progression, and responses in these patients were common (17\%, 20 of 121), highlighting atypical response kinetics. Therefore, in patients who are deriving clinical benefit despite imaging showing apparent progression, treatment beyond 
progression can be considered with careful discussion with the patient and quick confirmatory scans to rule out true progression. In patients with progressive disease and rapidly deteriorating physical condition, other treatment modalities and supportive care should be considered. Furthermore, patients who do not meet criteria for OR but have clinically meaningful stable disease should be continued on treatment. Future immunotherapy-based clinical trials should consider utilizing RECIST for exploratory response assessment to provide a standardized format for assessing atypical responses. ${ }^{46}$

\section{Duration of therapy}

At present, there are no guidelines to stop treatment if patients are deriving clinical benefit or have achieved a complete response. The financial burden of these agents, in addition to unexpected long-term AEs, should be considered and intermittent treatment further studied in clinical trials to minimize this burden.

\section{Role of biomarkers in patient selection for treatment with PDI/PDLI inhibitors PDLI expression}

Despite the durable responses observed across the clinical trials described in this review with PD1/PDL1 inhibitors, the majority of patients did not have an OR. This highlights the importance of identifying patients who may or may not respond to therapy. While all the clinical trials reviewed included exploratory assessment of PDL1 expression as a biomarker for response to PD1/PDL1 treatment, different PDL1-expression assays and antibodies were used for each PD1/PDL1 inhibitor (Table 3). Methodologies for staining, scoring, and threshold for positivity differed, and included assessment of PDL1 expression on tumor cells, stroma, and/or ICs. In addition, variability in timing of tissue collection and tissue-fixation and -processing methodologies between trials added to further differences. In addition to these technical factors, differences in the patient populations studied and pretreatment exposure could increase variability. With these limitations aside, no clear correlation can be observed between degree of PDL1 positivity and ORR (Figure 1). Across all the PDL1 thresholds utilized for positivity, a significant number of responses were observed below all the thresholds utilized. Therefore, the clinical utility of PDL1 expression at this time is limited to aiding patient education and shared decision-making, and should not be used to restrict access to treatment. Standardization and consensus are needed to harmonize PDL1 testing and interpretation.

\section{Tumor-mutation burden}

Tumor-mutation burden and neoantigen load was shown to correlate positively with response rates with atezolizumab (IMvigor 210, cohort 2) and avelumab (JAVELIN). Gene signatures, such as IFN $\gamma$ and basal subtype, were also shown to be predictive of response to nivolumab (CHECKMATE 275) and atezolizumab (IMvigor 210, cohort 2). These additional biomarkers could be potentially combined with PDL1 expression to improve selection of patients with a high likelihood of response to PD1/PDL1 inhibition.

\section{Human microbiome}

Some microbes in gut microbiota may facilitate treatment by enhancing host immunoresponse. A potential link between microbiota and response to checkpoint inhibitors in both mice and human patients has been reported. ${ }^{47}$ In animal models, mice with melanoma responded well to treatment with immunocheckpoint inhibitors as long as their microbiota was intact. However, if these mice have disturbed microbiota, immunocheckpoint inhibitors are no longer effective. The presence of certain bacteria in human microbiota (eg, commensal Bifidobacterium and Akkermansia muciniphila) is associated with an active immunoresponse when they are present in patients being treated with immunocheckpoint inhibitors. ${ }^{47,48}$ Absence of these activating microbiota may result in an ineffective response. Certain microbiota appear to protect and stimulate the immune system and allow immunocheckpoint inhibitors to have an antitumor response. Future studies will help assess the predictive role of the human microbiome in relation to response to PD1/PDL1 inhibitors.

\section{Future directions for pembrolizumab in UC}

There is a plethora of ongoing clinical trials with PD1/PDL1 inhibitors as single agents or in combination with other agents, eg, chemotherapy and immunotherapies, including vaccines, CTLA4 antibodies, LAG3, 4-1BB (CD137), IDO inhibitors, TIM3, CSF1, TNF-receptor superfamily member OX40, HDAC inhibitors, and enfortumab vedotin, in the metastatic setting. This is especially important, as currently we do not have a strategic approach for patients who progress on single-agent checkpoint inhibition, and developing novel, effective combinations with a checkpoint inhibitor as a backbone would be crucial to further development of immunotherapy to improve outcomes in bladder cancer. Besides the metastatic setting, there is a growing need to study the potential role of PD1/PDL1 inhibitors in NMIBC, MIBC, and neoadjuvant and adjuvant bladder cancer. 


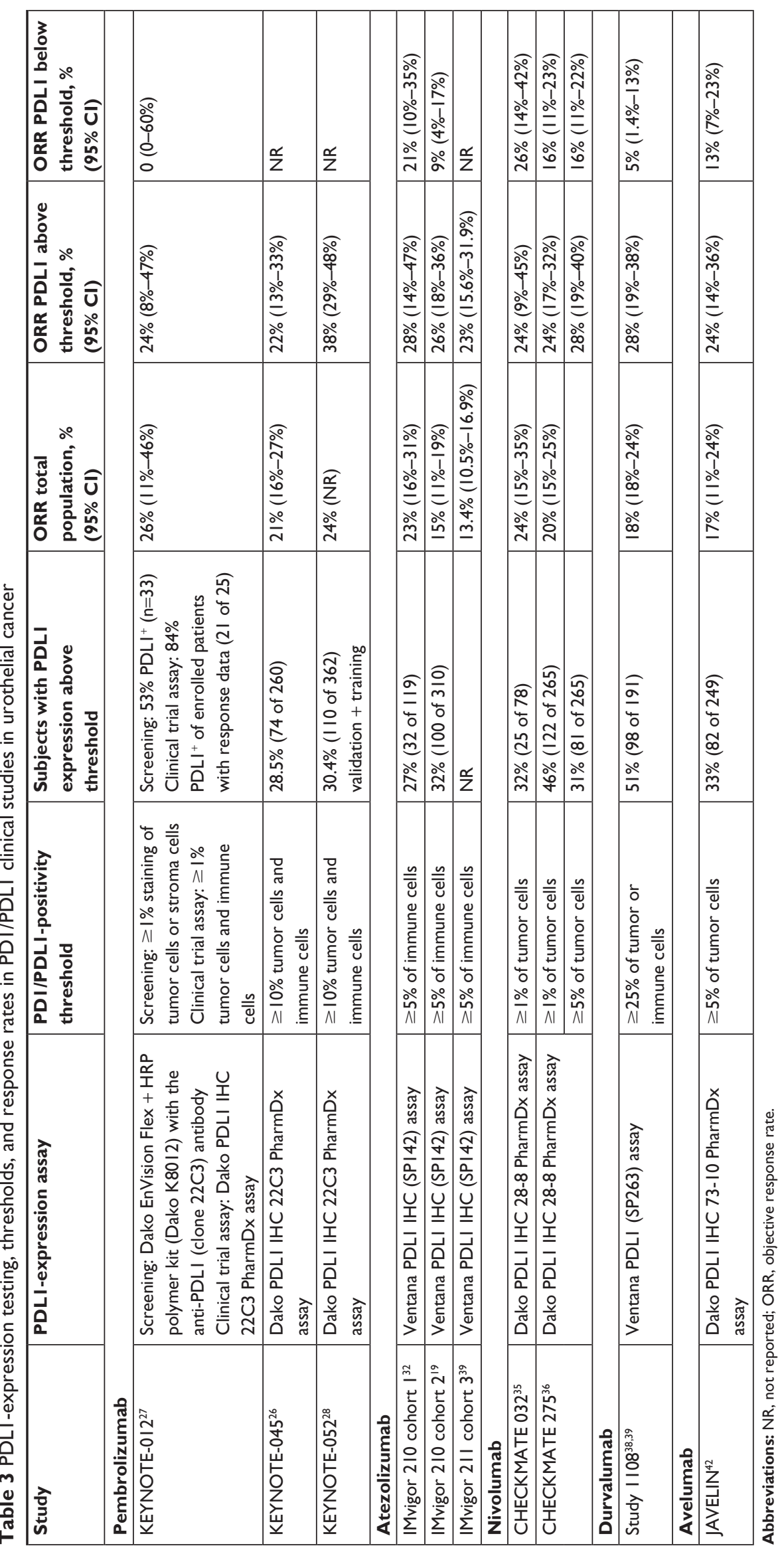




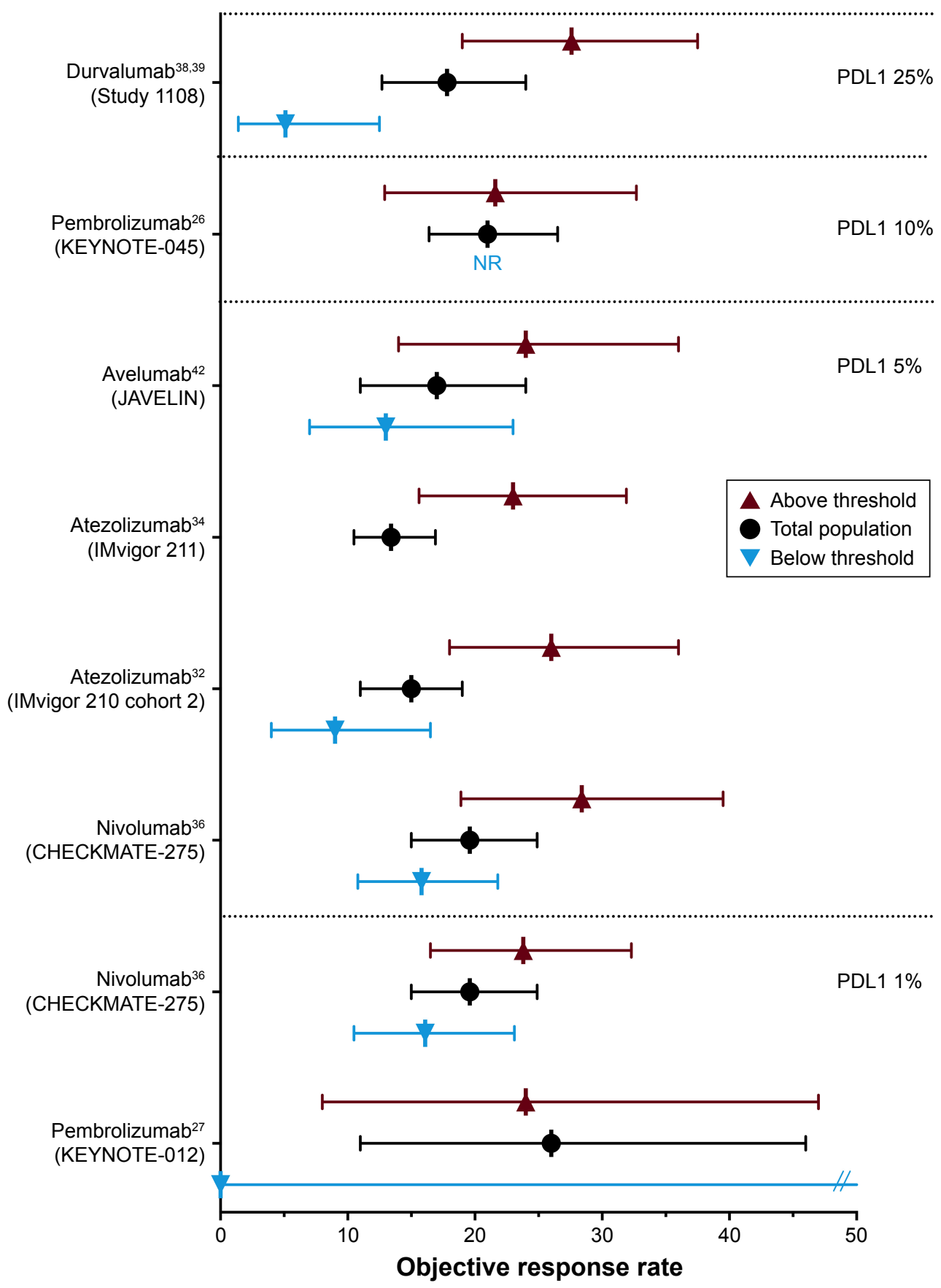

Figure I Objective response rate of PDI/PDLI inhibitors in the second-line setting.

Notes: For each study, the objective response rate and $95 \% \mathrm{Cl}$ are provided in the total population (black circle) and by PDLI expression above threshold (red upward triangle) and below threshold (blue downward triangles). Studies are ordered by PDLI expression thresholds.

\section{Non-muscle-invasive bladder cancer}

There are ongoing Phase II studies with pembrolizumab and atezolizumab in patients with high-risk BCG-refractory NMIBC (NCT02625961, NCT02844816). These studies present an alternative to radical cystectomy in patients who are not candidates for or are refusing cystectomy. There are trials exploring the safety of combination of PD1/PDL1 inhibitors and intravesical BCG in this setting. If encouraging, results from these trials would be crucial to move anti-PD1/ PDL1 agents to earlier in the course of UC paradigm and may radically change the current standard of care (cystectomy) for these patients by helping them preserve their bladders.

\section{Muscle-invasive bladder cancer}

In patients with MIBC, there are several ongoing studies evaluating the effect of single-agent neoadjuvant anti-PD1/PDL1 
agents and combinations with CTLA4 antibodies or chemotherapy on pathologic response rates (NCT02736266, NCT03294304, NCT03498196, NCT03234153, NCT02989584, and NCT02365766). Results from these trials will elucidate our understanding of whether checkpoint inhibitors can help maximize pathologic responses in MIBC, which may translate into survival benefit.

\section{Adjuvant setting in locally advanced bladder cancer}

There are several ongoing randomized trials comparing anti-PD1/PDL1 agents with placebo or observation in patients with MIBC and locally advanced bladder cancer (NCT03244384, NCT02632409, and NCT02450331). Over the coming years, data from these trials will help shed light on the role of adjuvant checkpoint inhibition, where currently there is no consensus on whether adjuvant therapy with chemotherapy should be offered or not.

\section{Concurrent use with radiation for bladder preservation}

A small ongoing Phase II study in New Zealand (NCT02662062) is studying the safety and best response achieved with use of pembrolizumab with concurrent radiation in patients planned for bladder preservation. Radiation therapy with immunotherapy is believed to stimulate the upregulation of immune molecules like PDL1 and PD1 to augment response to immunotherapy. ${ }^{41}$ Clinical trials in this setting will provide new insights in development of checkpoint inhibitors in these various stages of bladder cancer, with the goal of improving outcomes. In addition, biomarker correlatives from ongoing and planned studies should potentially help us select "responders" vs "nonresponders" and help us personalize the therapies we offer to patients, instead of offering a blanket checkpoint therapy to all patients.

\section{Conclusion}

After decades of stumbling blocks in drug development in bladder cancer, the advent of checkpoint immunotherapies has rapidly become an indispensable tool in our armamentarium against bladder cancer. Ongoing and planned studies with various checkpoint inhibitors alone or in combination with other agents across the various stages and lines of bladder cancer treatment will further advance the scope of these agents. While we cannot use a reliable biomarker to guide treatment decisions for our patients currently, the novel correlative studies in ongoing and planned trials will hopefully help select candidate biomarkers to optimize patient selection in future. Importantly, selecting patients who would not benefit from immunotherapy would not only avoid offering ineffective therapy but also avoid the significant financial toxicity of these agents unnecessarily. Lastly, immunotherapy has shown considerable promise in improving and maintaining the quality of life of patients suffering from bladder cancer, especially those with significant comorbidities and ineligible to receive standard therapies. In summary, immunotherapy will continue to shape the future bladder cancer paradigm, and the future is undoubtedly bright in terms of tackling disease after a long void.

\section{Author contributions}

DDS and SG were responsible for study conception/design and project supervision, DDS and DT for acquisition, analysis, or interpretation of data and table/figure drafting, DDS, SG, DT, and MAN for drafting the manuscript, and DDS, SG, and DT for critical revision of the draft.

\section{Disclosure}

DSS has received research funding from Bristol-Myers Squibb, Novartis, AstraZeneca, Bioverativ. DSS has acted as a consultant for Bristol-Myers Squibb, Salarius Pharmaceuticals, BetaCat Pharmaceuticals, and Glycosbio. SG has acted as a consultant or in an advisory role for Merck, Genentech, Exelixis, Pfizer, AstraZeneca, and Bristol-Myers Squibb. The authors report no other conflicts of interest in this work.

\section{References}

1. Surveillance, Epidemiology, and End Results Program. Cancer stat facts: bladder cancer. Available from: https://seer.cancer.gov/statfacts/html/ urinb.html. Accessed November 17, 2017.

2. Cookson MS, Herr HW, Zhang ZF, Soloway S, Sogani PC, Fair WR. The treated natural history of high risk superficial bladder cancer: 15 -year outcome. J Urol. 1997;158(1):62-67.

3. Clark P. NCCN clinical practice guidelines in oncology (NCCN guidelines): bladder cancer. Version 1. 2018. Available from: https://www.nccn.org/ professionals/physician_gls/pdf/bladder.pdf. Accessed on May 18, 2018.

4. Pectasides D, Pectasides M, Nikolaou M. Adjuvant and neoadjuvant chemotherapy in muscle invasive bladder cancer: literature review. Eur Urol. 2005;48(1):60-68

5. von der Maase H, Hansen SW, Roberts JT, et al. Gemcitabine and cisplatin versus methotrexate, vinblastine, doxorubicin, and cisplatin in advanced or metastatic bladder cancer: results of a large, randomized, multinational, multicenter, phase III study. J Clin Oncol. 2000;18(17): 3068-3077.

6. Sternberg CN, de Mulder PH, Schornagel JH, et al. Randomized phase III trial of high-dose-intensity methotrexate, vinblastine, doxorubicin, and cisplatin (MVAC) chemotherapy and recombinant human granulocyte colony-stimulating factor versus classic MVAC in advanced urothelial tract tumors: European Organization for Research and Treatment of Cancer Protocol no. 30924. J Clin Oncol. 2001;19(10):2638-2646.

7. Lorusso V, Pollera CF, Antimi M, et al. A phase II study of gemcitabine in patients with transitional cell carcinoma of the urinary tract previously treated with platinum. Eur J Cancer. 1998;34(8):1208-1212.

8. McCaffrey JA, Hilton S, Mazumdar M, et al. Phase II trial of docetaxel in patients with advanced or metastatic transitional-cell carcinoma. J Clin Oncol. 1997;15(5):1853-1857. 
9. Papamichael D, Gallagher CJ, Oliver RT, Johnson PW, Waxman J. Phase II study of paclitaxel in pretreated patients with locally advanced/ metastatic cancer of the bladder and ureter. Br J Cancer. 1997;75(4): 606-607.

10. Vaughn DJ, Broome CM, Hussain M, Gutheil JC, Markowitz AB. Phase II trial of weekly paclitaxel in patients with previously treated advanced urothelial cancer. J Clin Oncol. 2002;20(4):937-940.

11. Dash A, Galsky MD, Vickers AJ, et al. Impact of renal impairment on eligibility for adjuvant cisplatin-based chemotherapy in patients with urothelial carcinoma of the bladder. Cancer. 2006;107(3):506-513.

12. Galsky MD, Hahn NM, Rosenberg J, et al. Treatment of patients with metastatic urothelial cancer "unfit" for cisplatin-based chemotherapy. J Clin Oncol. 2011;29(17):2432-2438.

13. Bellmunt J, Mottet N, de Santis M. Urothelial carcinoma management in elderly or unfit patients. EJC Suppl. 2016;14(1):1-20.

14. Morales A, Eidinger D, Bruce AW. Intracavitary bacillus Calmette-Guérin in the treatment of superficial bladder tumors. J Urol. 1976;116(2): 180-182.

15. Morales A. Treatment of carcinoma in situ of the bladder with BCG. Cancer Immunol Immunother. 1980;9(1-2):69-72.

16. Alexandrov LB, Nik-Zainal S, Wedge DC, et al. Signatures of mutational processes in human cancer. Nature. 2013;500(7463): 415-421.

17. Snyder A, Makarov V, Merghoub T, et al. Genetic basis for clinical response to CTLA-4 blockade in melanoma. N Engl J Med. 2014; 371(23):2189-2199.

18. Rizvi NA, Hellmann MD, Snyder A, et al. Cancer immunology: mutational landscape determines sensitivity to PD-1 blockade in non-small cell lung cancer. Science. 2015;348(6230):124-128.

19. Rosenberg JE, Hoffman-Censits J, Powles T, et al. Atezolizumab in patients with locally advanced and metastatic urothelial carcinoma who have progressed following treatment with platinum-based chemotherapy: a single-arm, multicentre, phase 2 trial. Lancet. 2016; 387(10031):1909-1920.

20. Ribas A. Releasing the brakes on cancer immunotherapy. $N$ Engl J Med. 2015;373(16):1490-1492.

21. US Food and Drug Administration. Keytruda (pembrolizumab) [prescribing information]. 2016. Available from: https://www.accessdata. fda.gov/drugsatfda_docs/label/2016/125514s012lbl.pdf. Accessed September 8, 2017.

22. Puzanov I, Dummer R, Schachter J, et al. Efficacy based on tumor PD-L1 expression in KEYNOTE-002, a randomized comparison of pembrolizumab (pembro; MK-3475) versus chemotherapy in patients (pts) with ipilimumab-refractory (IPI-R) advanced melanoma (MEL). Journal of Clinical Oncology. 2015;33(15 suppl):3012-3012.

23. Schachter J, Ribas A, Long GV, et al. Pembrolizumab versus ipilimumab for advanced melanoma: final overall survival results of a multicentre, randomised, open-label phase 3 study (KEYNOTE-006). The Lancet. 2017;390(10105):1853-1862.

24. Herbst RS, Baas P, Kim D-W, et al. Pembrolizumab versus docetaxel for previously treated, PD-L1-positive, advanced non-small-cell lung cancer (KEYNOTE-010): a randomised controlled trial. The Lancet. 2016;387(10027):1540-1550.

25. Langer CJ, Gadgeel SM, Borghaei H, et al. Carboplatin and pemetrexed with or without pembrolizumab for advanced, non-squamous nonsmall-cell lung cancer: a randomised, phase 2 cohort of the open-label KEYNOTE-021 study. Lancet Oncol. 2016;17(11):1497-1508.

26. Bellmunt J, de Wit R, Vaughn DJ, et al. Pembrolizumab as Second-Line Therapy for Advanced Urothelial Carcinoma. New England Journal of Medicine. 2017;376(11):1015-1026.

27. Plimack ER, Bellmunt J, Gupta S, et al. Safety and activity of pembrolizumab in patients with locally advanced or metastatic urothelial cancer (KEYNOTE-012): a non-randomised, open-label, phase $1 \mathrm{~b}$ study. The Lancet Oncology. 2017;18(2):212-220.

28. Balar AV, Castellano D, O'Donnell PH, et al. First-line pembrolizumab in cisplatin-ineligible patients with locally advanced and unresectable or metastatic urothelial cancer (KEYNOTE-052): a multicentre, singlearm, phase 2 study. Lancet Oncol. 2017;18(11):1483-1492.
29. Moskowitz CH, Zinzani PL, Fanale MA, et al. Pembrolizumab in Relapsed/ Refractory Classical Hodgkin Lymphoma: Primary End Point Analysis of the Phase 2 Keynote-087 Study. Blood. 2016;128(22):1107-1107.

30. Sweeney CJ, Roth BJ, Kabbinavar FF, et al. Phase II study of pemetrexed for second-line treatment of transitional cell cancer of the urothelium. J Clin Oncol. 2006;24(21):3451-3457.

31. Harris J. Update sustains frontline pembrolizumab benefit in urothelial carcinoma. 2017. Available from: http:/www.onclive.com/ web-exclusives/update-sustains-frontline-pembrolizumab-benefit-inurothelial-carcinoma. Accessed January 23, 2018.

32. Balar AV, Galsky MD, Rosenberg JE, et al. Atezolizumab as first-line treatment in cisplatin-ineligible patients with locally advanced and metastatic urothelial carcinoma: a single-arm, multicentre, phase 2 trial. Lancet. 2017;389(10064):67-76.

33. Tecentriq (atezolizumab) [prescribing information]. 2018. Available from: https:/www.gene.com/download/pdf/tecentriq_prescribing.pdf. Accessed July 20, 2018

34. Powles T, Durán I, van der Heijden MS, et al. Atezolizumab versus chemotherapy in patients with platinum-treated locally advanced or metastatic urothelial carcinoma (IMvigor 211): a multicentre, open-label, phase 3 randomised controlled trial. Lancet. 2018;391(10122):748-757.

35. Sharma $\mathrm{P}$, Callahan MK, Bono $\mathrm{P}$, et al. Nivolumab monotherapy in recurrent metastatic urothelial carcinoma (CheckMate 032): a multicentre, open-label, two-stage, multi-arm, phase 1/2 trial. Lancet Oncol. 2016;17(11):1590-1598.

36. Sharma P, Retz M, Siefker-Radtke A, et al. Nivolumab in metastatic urothelial carcinoma after platinum therapy (CheckMate 275): a multicentre, single-arm, phase 2 trial. Lancet Oncol. 2017;18(3):312-322.

37. AstraZeneca. Imfinzi (durvalumab) [highlights of prescribing information]. 2017. Available from: https:/www.azpicentral.com/imfinzi/ imfinzi.pdf. Accessed May 1, 2017.

38. Powles T, O’Donnell PH, Massard C, et al. Efficacy and safety of durvalumab in locally advanced or metastatic urothelial carcinoma: updated results from a phase 1/2 open-label study. JAMA Oncol. 2017;3(9):e172411.

39. Massard C, Gordon MS, Sharma S, et al. Safety and efficacy of durvalumab (MEDI4736), an anti-programmed cell death ligand-1 immune checkpoint inhibitor, in patients with advanced urothelial bladder cancer. J Clin Oncol. 2016;34(26):3119-3125.

40. US Food and Drug Administration. Bavencio (avelumab) [prescribing information]. 2017. Available from: https://www.accessdata.fda.gov/drugsatfda_docs/label/2017/761049s000lbl.pdf. Accessed July 20, 2018.

41. Apolo AB, Infante JR, Balmanoukian A, et al. Avelumab, an antiprogrammed death-ligand 1 antibody, in patients with refractory metastatic urothelial carcinoma: results from a multicenter, phase IB study. J Clin Oncol. 2017;35(19):2117-2124.

42. Patel MR, Ellerton J, Infante JR, et al. Avelumab in metastatic urothelial carcinoma after platinum failure (JAVELIN solid tumor): pooled results from two expansion cohorts of an open-label, phase 1 trial. Lancet Oncol. 2018;19(1):51-64.

43. Puzanov I, Diab A, Abdallah K, et al. Managing toxicities associated with immune checkpoint inhibitors: consensus recommendations from the Society for Immunotherapy of Cancer (SITC) Toxicity Management Working Group. J Immunother Cancer. 2017;5(1):95.

44. Champiat S, Lambotte O, Barreau E, et al. Management of immune checkpoint blockade dysimmune toxicities: a collaborative position paper. Ann Oncol. 2016;27(4):559-574.

45. Haanen J, Carbonnel F, Robert C, et al. Management of toxicities from immunotherapy: ESMO clinical practice guidelines for diagnosis, treatment and follow-up. Ann Oncol. 2017;28(Supp1 4):iv119-iv142.

46. Seymour L, Bogaerts J, Perrone A, et al. iRECIST: guidelines for response criteria for use in trials testing immunotherapeutics. Lancet Oncol. 2017;18(3):e143-e152.

47. Routy B, le Chatelier E, Derosa L, et al. Gut microbiome influences efficacy of PD-1-based immunotherapy against epithelial tumors. Science. 2018;359(6371):91-97.

48. Sivan A, Corrales L, Hubert N, et al. Commensal Bifidobacterium promotes antitumor immunity and facilitates anti-PD-L1 efficacy. Science. 2015;350(6264):1084-1089. 
OncoTargets and Therapy

\section{Publish your work in this journal}

OncoTargets and Therapy is an international, peer-reviewed, open access journal focusing on the pathological basis of all cancers, potential targets for therapy and treatment protocols employed to improve the management of cancer patients. The journal also focuses on the impact of management programs and new therapeutic agents and protocols on

patient perspectives such as quality of life, adherence and satisfaction. The manuscript management system is completely online and includes a very quick and fair peer-review system, which is all easy to use. Visit http://www.dovepress.com/testimonials.php to read real quotes from published authors.

Submit your manuscript here: http://www.dovepress.com/oncotargets-and-therapy-journal 